\title{
Application of the International System for Reporting Serous Fluid Cytopathology (ISRSFC) on Reporting Pericardial Effusion Cytology
}

\author{
Erika F. Rodriguez ${ }^{a}$ Robert Jones ${ }^{a}$ Matthew Gabrielson ${ }^{a}$ Dustin Santos ${ }^{a}$ \\ Ricardo G. Pastorello ${ }^{b, c}$ Zahra Maleki ${ }^{a}$ \\ aDepartment of Pathology, Division of Cytopathology, The Johns Hopkins Hospital, Baltimore, MD, USA; \\ bepartment of Pathology, Division of Cytopathology, A.C. Camargo Cancer Center, São Paulo, Brazil; \\ 'Dana Farber/Brigham and Women's Cancer Center, Department of Pathology, Boston, MA, USA
}

\section{Keywords}

The International System for Reporting Serous Fluid Cytopathology · Pericardial effusions · Pericardial fluid . Effusion cytology $\cdot$ Nondiagnostic cases $\cdot$ Negative for malignancy · Atypia of undetermined significance .

Suspicious for malignancy · Malignant

\begin{abstract}
Introduction: The International System for Reporting Serous Fluid Cytopathology (ISRSFC) has recently been announced. Pericardial effusion (PE) is a clinical manifestation of a large variety of both neoplastic and non-neoplastic conditions. Herein, we have applied the ISRSFC on reporting PE cytopathology and report our experience in a large academic institution. Method and Materials: After the Institutional Research Board approval, the electronic pathology database of a large academic institution was queried for PEs collected from January 2014 to January 2019. The diagnosis, patient demographics, and specimen volume were recorded for each case. The ISRSFC was applied and the cases were divided into 5 categories: nondiagnostic (ND), negative for
\end{abstract}

malignancy (NFM), atypia of uncertain significance (AUS), suspicious for malignancy (SFM), and malignant (MAL). Each category was evaluated separately. Results: A total of 299 cases were identified, 162 females and 137 males. The age of the subjects ranged from less than a year to 89 years (average 51.25 years). The volume ranged from 3 to $1,700 \mathrm{~mL}$ (average $298 \mathrm{~mL}$ ). There were 252 NFM (84.3\%), 13 AUS (4.3\%), 4 SFM (1.3\%), and 30 MAL (10\%) cases. Metastatic lung cancer followed by metastatic breast cancer were the most common malignancies involving pericardial fluid (PF). No cases were diagnosed as ND. However, no mesothelial cells were seen in 97 specimens (38\% of the negative cases). None of these patients developed malignant PE in at least 6 months of follow-up. Conclusion: The ISRSFC is a user-friendly reporting system which is easily applicable on serous fluid including PF. The vast majority of PEs was benign (84.3\%). Our study shows that the presence of mesothelial cells is not necessary for specimen adequacy in serous effusions as no mesothelial cells were identified in $38 \%$ of the negative cases. Metastatic lung carcinoma was the most common diagnosis of malignant effusions.

(c) 2020 S. Karger AG, Basel

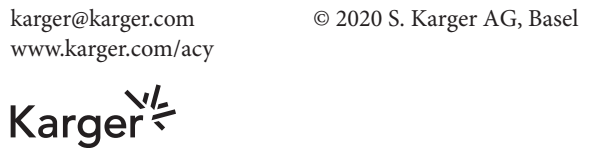




\section{Introduction}

Serous cavity effusions comprise a large number of cytology specimens in every cytopathology practicing setup. Serous fluid accumulation in the pleural cavity, pericardium, and peritoneal/abdominal/pelvic cavity is a clinical presentation of variable pathologic causes and subsequently variable cellular components. Serous fluid cytopathology may possess diagnostic challenges due to marked reactive atypia of mesothelial cells, cytomorphologic overlap of malignant cells and mesothelial cells, or simply the presence of benign glandular cells. Therefore, reporting cytopathologic findings of these specimens plays a crucial role in patients' clinical management. However, there is no universal system of reporting serous fluid cytopathology specimens. In the footsteps of prior established reporting systems such as the Bethesda System (TBS) for reporting Cervical Cytology, the Bethesda System for Reporting Thyroid Cytopathology (BethesdaThyroid), the Paris System (TPS) for Reporting Urinary Cytology, and the Milan system for Reporting Salivary Gland Cytopathology (MSRSGC), an initiative was named "The International System for Reporting Serous Fluid Cytopathology (ISRSFC)" and established to create a reporting system for serous fluid cytopathology, and it was endorsed by the International Academy of Cytology (IAC) and the American Society of Cytopathology (ASC) [1-4].

The aim of the ISRSFC is to develop an evidencedbased diagnostic system along with management recommendation in order to enhance professional communication and improve patient care. The ISRSFC is a five-tiered diagnostic category including nondiagnostic (ND), negative for malignancy (NFM), atypia of undetermined significance (AUS), suspicious for malignancy (SFM), and malignant (MAL) - primary and secondary [5]. Herein we have applied the ISRSFC on pericardial effusion (PE) cytopathology cases and report our findings and the significance of each category.

The pericardium is a double-layered fibroelastic sac that envelops the heart and great vessels. It is formed by the visceral pericardium composed of a delicate mesothelial monolayer and by the parietal pericardium. The space between the visceral and parietal pericardium is called pericardial space, and it holds a thin layer of pericardial fluid (PF). PEs are characterized by the accumulation of fluid in the pericardial space above the normally seen 20 $60 \mathrm{~mL}$ of fluid in the pericardial space [6], which can ultimately compress heart structures and impair function, leading to cardiac tamponade.
Several etiologic factors can cause PE, including viral or tuberculous infection, radiation, post-cardiac injury syndrome, certain medications, metabolic abnormalities (such as uremia), collagen vascular disease, idiopathic or immune-mediated inflammatory disorders, and malignancy [7-9].

Malignancy is an important cause of PE. Malignant involvement of the pericardium has been reported in $2-31 \%$ of cancer-related autopsies. It can develop secondary to direct extension of the malignant neoplasm or metastatic disease $[10,11]$. The malignant process may be due to solid tumors such as lung, breast, and esophageal cancer, or due to hematologic malignancies $[10,11]$.

Cytologic evaluation is usually performed in all patients undergoing pericardiocentesis for treatment of cardiac tamponade, including in those with suspected infectious or malignant pericarditis [12]. Although cytology is part of the evaluation of $\mathrm{PE}$, a definitive diagnosis following pericadiocentesis is achieved in less than $40 \%$ of cases $[7,8]$. Herein, we report our experience with application of the ISRSFC on reporting PE cytopathology.

\section{Material and Methods}

\section{Patient Selection}

After Institutional Review Board (IRB) approval, the electronic pathology database at the Johns Hopkins Hospital was queried for PE specimens collected from January 2014 to January 2019. Patient demographics, cytologic diagnosis, ancillary studies, past medical history, comorbid conditions, and the volume of pericardial specimen were recorded for each case. The slides including immunohistochemical stains and the cell blocks of all cases were re-reviewed by 2 board-certified pathologists (E.R. and Z.M.) to verify the diagnosis. The ISRSFC was applied and the cases were divided into 5 categories: ND, NFM, AUS, SFM, and MAL. Each category was evaluated separately. The NFM category was divided into 11 categories based upon their possible risk factors for PE, including uremia due to acute and chronic renal failure; connective tissue disorders; hypothyroidism; anticoagulant therapy; non-anticoagulant medications; cardiovascular conditions such as post-cardiac surgery, myocardial infarction, or ablation procedures; infections; radiation therapy; trauma; organ transplant; and unknown etiology. The cellular component of each category was recorded.

\section{Criteria Used for Each ISRSFC Category}

ND: This category consists of extremely scant specimens with no cells or rare benign-appearing cells (usually less than 10 cells) such as a few macrophages or lymphocytes or mesothelial cells or RBCs.

NFM: Specimens consist of one or more cell types of benignappearing mesothelial cells, lymphocytes, blood, and macrophages.

AUS: This category is used when (1) there are a few atypical cells of undetermined origin, or (2) there are atypical lymphocytes, or (3) there are atypical mesothelial cells. 
Table 1. Patients' demographics, diagnostic categories, and specimen volume

\begin{tabular}{|c|c|c|c|c|}
\hline $\begin{array}{l}\text { Diagnostic } \\
\text { category }\end{array}$ & Gender & $\begin{array}{l}\text { Age, } \\
\text { years }\end{array}$ & $\begin{array}{l}\text { Volume, } \\
\mathrm{mL}\end{array}$ & $\begin{array}{l}\text { Ancillary } \\
\text { studies }\end{array}$ \\
\hline Benign $(n=252)$ & $\begin{array}{l}\text { M: } 120 \\
\text { F: } 132\end{array}$ & $\begin{array}{l}\text { Min: }<1 \\
\text { Max: } 89 \\
\text { Ave: } 54.2\end{array}$ & $\begin{array}{l}\text { Min: } 3 \\
\text { Max: } 1,700 \\
\text { Ave: } 300.3\end{array}$ & $\begin{array}{l}\mathrm{IHC}=26 \\
\text { Flow }=2\end{array}$ \\
\hline Atypical $(n=13)$ & $\begin{array}{l}\text { M: } 6 \\
F: 7\end{array}$ & $\begin{array}{l}\text { Min: } 22 \\
\text { Max: } 85 \\
\text { Ave: } 54.5\end{array}$ & $\begin{array}{l}\text { Min: } 6 \\
\text { Max: } 400 \\
\text { Ave: } 207\end{array}$ & $\begin{array}{l}\text { IHC }=7 \\
\text { Flow }=1\end{array}$ \\
\hline Suspicious $(n=4)$ & F: 4 & $\begin{array}{l}\text { Min: } 38 \\
\text { Max: } 86 \\
\text { Ave: } 59\end{array}$ & $\begin{array}{l}\text { Min: } 6 \\
\text { Max: } 800 \\
\text { Ave: } 427\end{array}$ & $\mathrm{IHC}=3$ \\
\hline $\operatorname{MAL}(n=30)$ & $\begin{array}{l}\text { M: } 6 \\
\text { F: } 24\end{array}$ & $\begin{array}{l}\text { Min: } 14 \\
\text { Max: } 87 \\
\text { Ave: } 52.3\end{array}$ & $\begin{array}{l}\text { Min: } 8 \\
\text { Max: } 800 \\
\text { Ave: } 307\end{array}$ & $\mathrm{IHC}=15$ \\
\hline Total $(n=299)$ & $\begin{array}{l}\text { M: } 136 \\
\text { F: } 163\end{array}$ & $\begin{array}{l}\text { Min: }<1 \\
\text { Max: } 89 \\
\text { Ave: } 51.25\end{array}$ & $\begin{array}{l}\text { Min: } 3 \\
\text { Max: } 1,700 \\
\text { Ave: } 299\end{array}$ & $\begin{array}{l}\mathrm{IHC}=51 \\
\text { Flow }=3\end{array}$ \\
\hline
\end{tabular}

M, male; F, female; Min, minimum; Max, maximum; Ave, average; IHC, immunohistochemistry; MAL, malignant.

SFM: This category is used when there are (1) recognizable cell types of markedly atypical epithelial cells, or (2) markedly atypical lymphocytes, (3) markedly atypical mesothelial cells, or (4) any markedly atypical cells suspicious for a specific type of malignancy, for instance suspicious for melanoma. However, there are insufficient malignant cells for further characterization of malignant cells through ancillary studies such as immunohistochemistry (IHC) or flow cytometry.

MAL - primary and secondary: Any type of malignancy is included in this category.

\section{Preparation Method}

Specimens were received fresh and prepared according to standard clinical processing. The PE preparation method is the same as that used for effusions and is detailed in other publications [13-15]. In summary, effusions were either entirely submitted for centrifugation or a representative $100-\mathrm{mL}$ sample was processed. During processing, specimens were divided into 2 tubes and centrifuged. One of the tubes was used for preparing 2 slides using the cytospin method (Thermo Fisher Scientific, Waltham, MA, USA) and stained with the Papanicolaou stain. A cell block is routinely prepared for all samples unless there is inadequate material. Occasionally, a second cell block might be prepared if it is indicated, such as additional material for molecular studies. A cell pellet was obtained from the other tube and the material was fixed in formalin, processed as a cell block, and stained with hematoxylin and eosin. Immunohistochemical staining was performed at the time of diagnosis on the cell block, according to clinical protocols validated in our immunohistochemical laboratory.

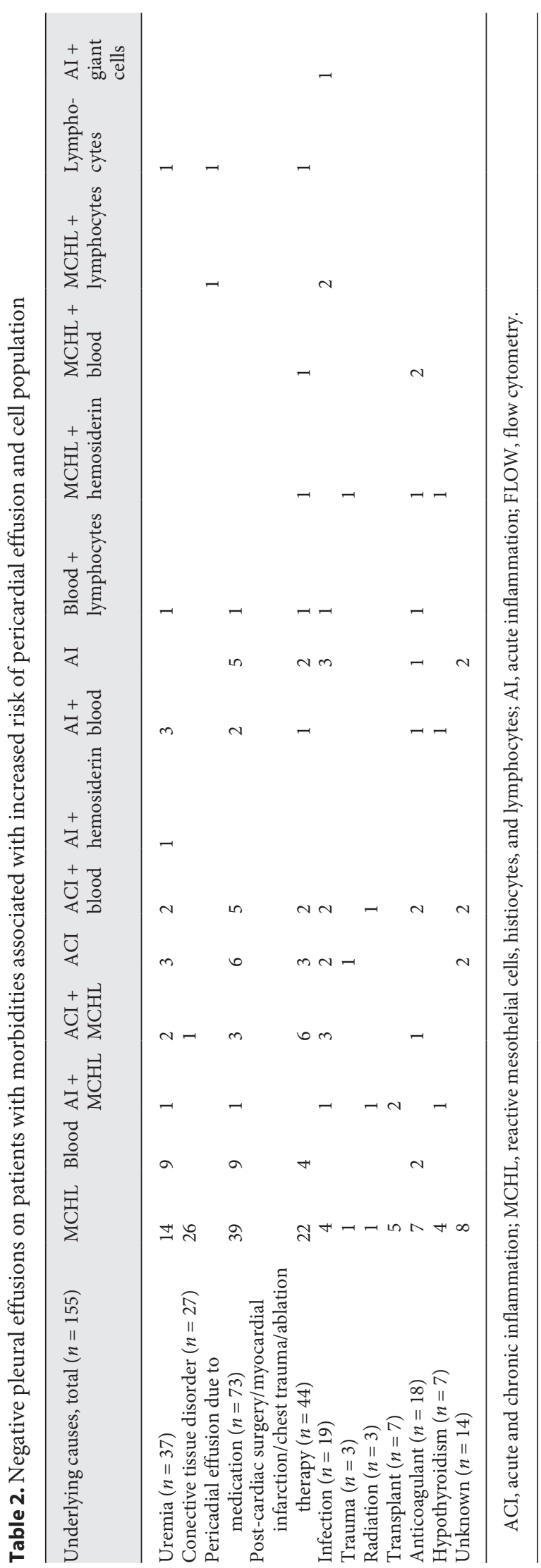

Acta Cytologica 2020;64:477-485 


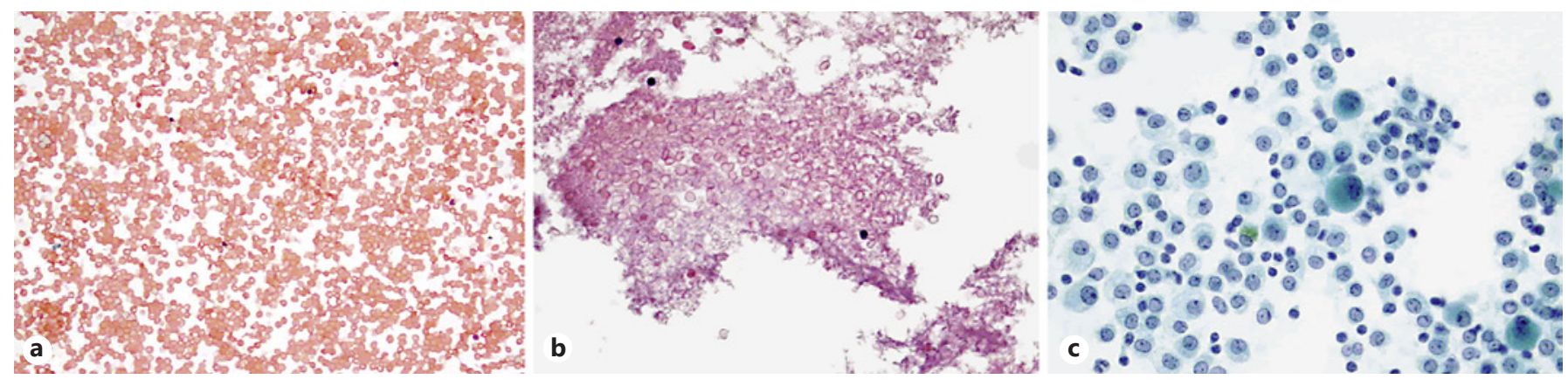

Fig. 1. a NFM, pleural effusion consists of blood only ( $\times 200$, Diff-Quik stain). b NFM, a cell block preparation of pleural effusion consists of blood only $(\times 100$, H\&E stain). c NFM, pleural fluid consists of mesothelial cells, lymphocytes, and macrophages. One hemosiderin-laden macrophage is seen $(\times 200$, Papanicolaou stain). NFM, negative for malignancy; $\mathrm{H} \& \mathrm{E}$, hematoxylin and eosin.

\section{Results}

In total, cytology reports from 299 pericardiocentesis performed within a 5-year period were included in the study (Table 1). Patients were predominantly female $(n=$ $162,64.2 \%)$. The mean age of the study group was 54 years (range $0-89$ years). The majority of the study population identified themselves as white $(n=158,52.8 \%)$, followed by black ( $n=91,30.4 \%)$, other $(n=25,8.4 \%)$, Asian $(n=16,5.4 \%)$, unknown $(n=6,2 \%)$, indigenous $(n=2$, $0.7 \%)$ and mixed race $(n=1,0.3 \%)$. A previous history of malignancy was noted in $137(45.8 \%)$ patients. Of all specimens, $252(84.3 \%)$ were diagnosed as NFM, 13 $(4.3 \%)$ as AUS, $4(1.3 \%)$ as SFM, and $30(10 \%)$ as MAL. Ancillary IHC and flow cytometric studies were performed in a minority of cases $(n=28,9.4 \%$ and $n=5$, $1.7 \%$, respectively).

\section{Negative for Malignancy}

Patients with a NFM diagnosis on cytology $(n=252)$ were predominantly female $(n=131,52 \%)$, with a mean age of 54 years. The mean volume of these samples was $299 \mathrm{~mL}$ (range 3-1,700 mL). The majority of the patients had no previous history of malignancy $(n=151,59.9 \%)$. Of the clinically suspected causes for the PEs, most were unknown or not specified ( $n=123,48.8 \%$ ), followed by NFM $(n=100,39.7 \%)$ and MAL $(n=29,11.5 \%)$. One hundred fifty-five patients had history of known risk factors for PE (Table 2). Reactive mesothelial cells, histiocytes, and lymphocytes ( $n=72,28.6 \%)$ was the most frequent cellular component found (Fig 1). Infection was considered the main underlying cause of PE in 10 cases. The infectious organisms were as follows: Propionibacterium acnes $(n=3)$, Propionibacterium acnes and Strepto- coccus viridans $(n=1)$, Streptococcus pneumoniae $(n=2)$, methicillin-resistant Staphylococcus aureus $(n=2)$, coxsackie B virus $(n=1)$, and Trypanosoma brucei $(n=1)$.

NFM Specimens where Mesothelial Cells Were Absent

In 97 specimens (33\% of the total number of 252 cases $/ 38 \%$ of the negative cases), mesothelial cells were not present. Of those 97 specimens, 40 (41\%) had a history of malignancy. However, only 7 of the 40 (18\%) specimens had a clinical SFM PE. For 49 of the 97 specimens, the clinically suspected cause was unknown on chart review. None of the patients with specimens signed as NFM on cytology but without mesothelial cells present on specimens developed malignant $\mathrm{PE}$ in at least 8 months followup. The cytologic components for this category were variable, including blood only $(26,10.3 \%)$, blood and lymphocytes $(5,2 \%)$, blood and acute inflammatory cells $(8$, $3.2 \%)$, blood and mixed inflammatory cells $(19,8 \%)$, acute inflammatory cells $(16,6.3 \%)$, acute inflammatory cells and hemosiderin pigment $(1,0.4 \%)$, acute inflammatory cells and giant cells $(1,0.4 \%)$, acute and chronic inflammatory cells $(18,7.1 \%)$, and lymphocytes $(3,1 \%)$. The diagnosis was reached and verified by reviewing 2 cytospin slides and the cell blocks. No additional IHC was performed to detect mesothelial cells. It is neither costeffective nor informative to perform immunohistochemical staining on blood-only specimens.

\section{Atypia of Undetermined Significance}

Of the 13 cytology specimens diagnosed as "AUS," most were from female patients $(n=7,53.8 \%)$, with a mean age of 52 years. The mean volume of these samples was $175.5 \mathrm{~mL}$ (range 6-500 mL). Majority of the patients had a previous history of malignancy $(n=9,69.2 \%)$, in- 


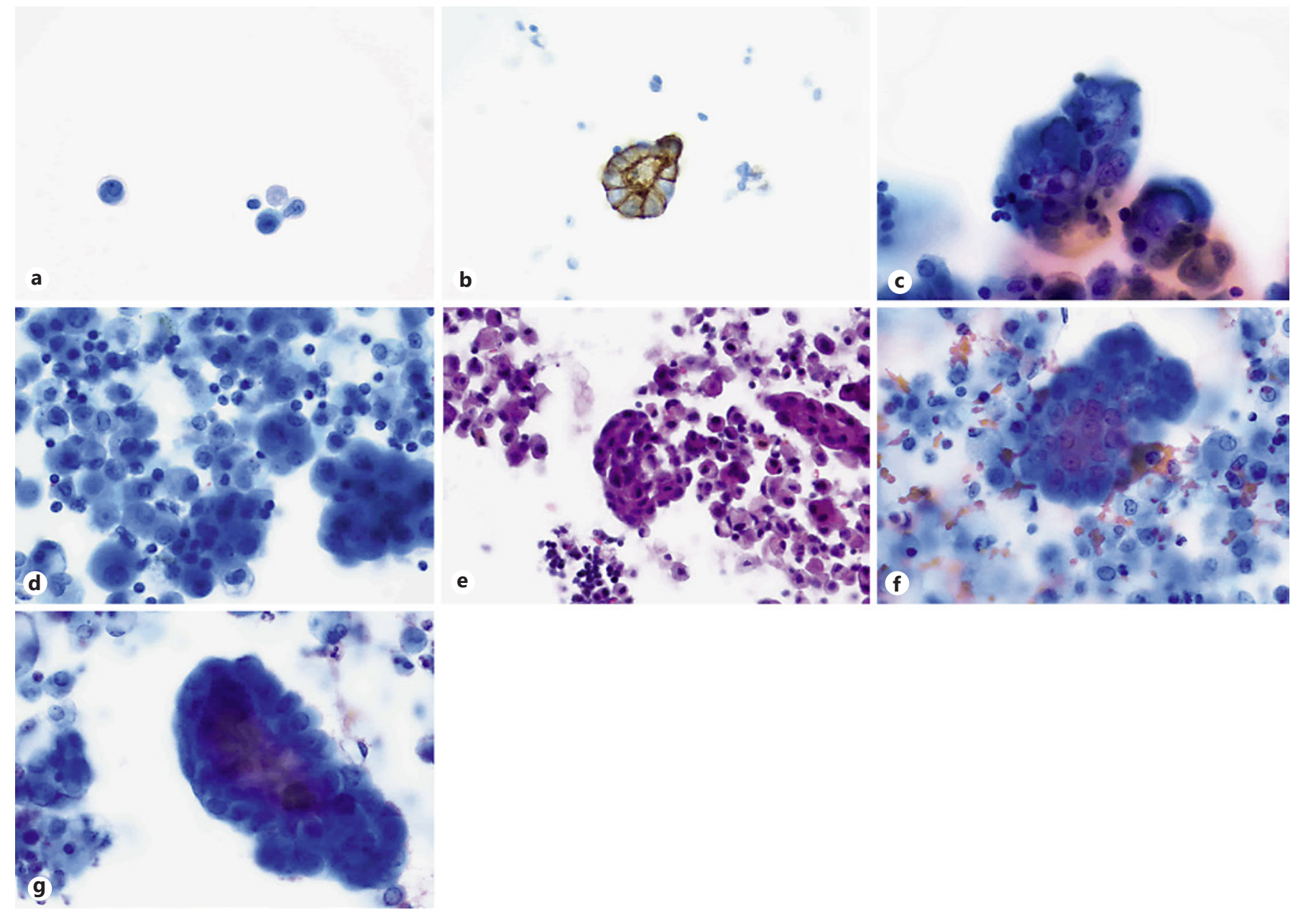

Fig. 2. a AUS, rare atypical epithelioid cells are seen characterized by high nuclear to cytoplasmic ratio, irregular nuclear membrane, and prominent nucleoli ( $\times 200$, Papanicolaou stain). b AUS, BEREP4 immunostain highlights one cluster of epithelial cells $(\times 200$, immunostain). c, d AUS, clusters of atypical epithelioid cells are seen in a background of mesothelial cells, macrophages, and lymphocytes ( $\times 200$, Papanicolaou stain). e AUS, a few clusters of epi-

cluding $4(44.4 \%)$ carcinomas, $4(44.4 \%)$ hematologic malignancies, and 1 (11.1\%) sarcoma. Clinical suspicion of the origin of the effusions was mainly unknown/not specified $(n=11,84.6 \%)$, followed by malignant and nonmalignant (both $n=1,7.7 \%$ ). The atypia present was predominantly of epithelial and hematopoietic origin (both $n=6,46.2 \%)$, followed by mesothelial origin ( $n=1,7.7 \%)$. Ancillary studies were attempted, including IHC in the majority of the atypical cases $(n=10,76.9 \%)$ and flow cytometry in 3 samples (23.1\%) (Fig. 2).

Based on patients' prior history of malignancy, immunohistochemical stainings were performed to further characterize the nature of the atypical cells. BEREP-4 thelioid cells are seen on a cell block preparation $(\times 100, H \& E)$. The immunohistochemical stains were noncontributory. f, g SFM, a cluster of markedly atypical epithelial cells is seen ( $\times 200$, Papanicolaou stain). Higher magnification shows prominent nucleoli of the cells ( $\times 400$, Papanicolaou stain). AUS, atypia of undetermined significance; H\&E, hematoxylin and eosin; SFM, suspicious for malignancy. $(n=7$, positive $=5$, negative $=1$, and inconclusive $=1)$ and calretinin ( $n=6$, positive $=3$, negative $=2$, and inconclusive $=1$ ) stainings were performed to differentiate atypical epithelial cells versus reactive mesothelial cells, respectively. Thyroid transcription factor-1 (TTF-1) $(n=$ 3 , positive $=1$, negative $=1$, and inconclusive $=1$ ) and napsin $\mathrm{A}(n=2$, positive $=1$ and inconclusive $=1)$ were performed to confirm a metastatic lung adenocarcinoma; GATA binding protein 3 (GATA-3) $(n=2$, positive $=1$ and inconclusive $=1)$, mammoglobin $(n=2$, both negative), and estrogen receptor (ER) ( $n=2$, both negative) for a breast primary; caudal type homeobox 2 (CDX-2) $(n=2$, negative $=1$ and inconclusive $=1)$ and cytokeratin 


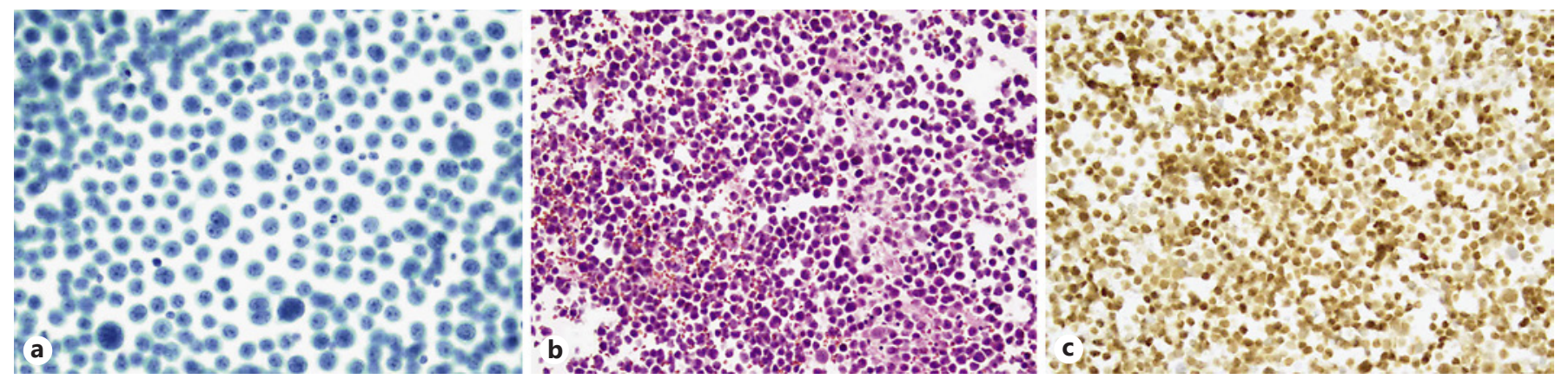

Fig. 3. a MAL, metastatic adenocarcinoma of a lung primary involving the pericardial space manifested by numerous malignant single cells $(\times 200$, Papanicolaou stain). b A cell block preparation showing numerous malignant cells and foci of necrosis $(\times 100, \mathrm{H} \& \mathrm{E}$ stain). c TTF- 1 immunostain showing nuclear staining of the malignant cells $(\times 200$, immunostain). MAL, malignant; H\&E, hematoxylin and eosin.

20 (CK20) ( $n=2$, both negative) for a colon primary; insolinoma-associated 1 (INSM1) ( $n=1$, negative) for a neuroendocrine tumor; and cluster of differentiation 68 (CD68) ( $n=1$, negative) to differentiate macrophages versus atypical epithelial cells. CD3 $(n=4$, positive $=2$, negative $=1$, and inconclusive $=1)$, CD20 $(n=4$, positive $=1$, negative $=2$, and inconclusive $=1$ ), and EpsteinBarr encoding region-in situ hybridization (EBER-ISH) $(n=1)$ were ordered to detect a mixed T-cell and B-cell population of lymphocytes versus a monoclonal process, and $\operatorname{CD} 15(n=2$, negative $=1$ and inconclusive $=1)$ and CD30 ( $n=2$, both inconclusive) for Hodgkin's disease. Paired box 8 (PAX8) ( $n=1$, inconclusive) and CK7 ( $n=1$, positive) were examined for a gynecologic primary malignancy. Desmin $(n=1)$ and KI-67 $(n=1)$ were inconclusive in one case with a history of uterine leiomyosarcoma.

\section{Suspicious for Malignancy}

The 4 samples deemed as SFM were mainly from male patients $(n=3,75 \%)$, with a mean age of 58 years, and all had a previous history of malignancy, 2 (50\%) had carcinomas, and $2(50 \%)$ were of hematopoietic origin. Half of the cases $(n=2,50 \%)$ had a nonmalignant clinical suspicion of the origin of the effusion, while $1(25 \%)$ was thought to be malignant and 1 (25\%) was unknown/not specified. The mean volume of these samples was $439 \mathrm{~mL}$ (range 6-800 mL). Half $(n=2,50 \%)$ were deemed suspicious due to cells of epithelial origin and the other half due to cells of hematopoietic origin. The majority $(n=3$, $75 \%$ ) of the cases had IHC attempted, while flow cytometry was performed in only one case (25\%).

The following stains were performed and the findings were as follows: BEREP-4 ( $n=1$, positive), calretinin $(n=$ 3 , negative $=2$ and inconclusive $=1), \operatorname{CD} 34(n=1$, posi- tive), and CD5 ( $n=1$, negative). GATA-3 $(n=1)$, mamoglobin $(n=1), \operatorname{Ki}-67(n=1), \operatorname{PR}(n=1)$, and ER $(n=1)$ were inconclusive in a patient with a history of breast cancer. S-100 $(n=1)$, calponin $(n=1)$, and smooth muscle actin (SMA) $(n=1)$ were focally positive.

\section{MAL - Primary and Secondary}

Patients with an MAL diagnosis on cytology $(n=30)$ were predominantly female $(n=23,76.7 \%)$, with a mean age of 51 years. All patients had a prior diagnosis of malignancy in their chart. However, a prior history of malignancy was provided by the clinician in only $23(76.7 \%)$ cases. The primary sites were the lungs ( $n=11,36.7 \%)$, breast $(n=7,23.3 \%)$, colon $(n=2,6.7 \%)$, stomach $(n=2$, $6.7 \%)$, and one $(3.3 \%)$ of each of the following: nuclear protein in testis (NUT) carcinoma, ovarian serous carcinoma, and renal cell carcinoma. Of the hematopoietic malignancies, 2 (50\%) were acute lymphoblastic leukemia and one case each (25\%) of multiple myeloma and T-cell lymphoma. The only noted sarcoma was a case of rhabdomyosarcoma in a 14-year-old male. The majority ( $n=18,60 \%)$ of cases had an unknown/not specified clinically suspected cause for the effusion in their charts, while $11(36.7 \%)$ were thought to be of malignant origin and $1(3.3 \%)$ was thought to be nonmalignant. The mean volume of these samples was $320.2 \mathrm{~mL}$ (range 5-900 mL). Half ( $n=15,50 \%)$ had IHC attempted, while flow cytometry was performed in only one case (3.3\%) (Fig. 3).

The staining results were as follows: BEREP-4 $(n=11$, positive $=11$ ) to detect malignant epithelial cells and calretinin $(n=10$, negative $=10)$ to detect atypical mesothelial cells. Immunohistochemical stains were utilized to confirm a primary site, including GATA-3 $(n=5$, positive $=2$ and negative $=3)$, TTF- $1(n=5$, positive $=2$ and 
negative $=3)$, napsin $\mathrm{A}(n=4$, positive $=1$ and negative $=3), \mathrm{P} 40(n=5$, focally positive $=1$ and negative $=$ $4)$, synaptophysin $(n=2$, positive $=1$ and negative $=1)$, and $\operatorname{CDX}-2(n=3$, positive $=1$ and negative $=2)$. Positive stains included desmin $(n=1)$, INSM-1 $(n=1)$, CK7 $(n=3), \mathrm{CD} 56(n=1), \mathrm{CD} 138(n=1), \mathrm{P} 53(n=2), \mathrm{CD} 435$ $(n=1)$, NUT $(n=1)$, and myogenin $(n=1)$. KI-67 was performed in 2 cases and showed a proliferation index of $70 \%$ and $50-80 \%$. The following stains were negative: CD68 $(n=1)$, CK20 $(n=3), \operatorname{PAX}-8(n=3), \mathrm{ER}(n=3)$, mamoglobulin $(n=2)$, PR $(n=1), \mathrm{S}-100(n=1)$, gross cystic disease fluid protein (GCDFP) $(n=1)$, Wilms' tumor 1 (WT-1) $(n=1)$, chromogranin $(n=1)$, human melanoma black 45 (HMB45) $(n=1)$, melan-A $(n=1)$, anti-cytokeratin 1 (AE1)/AE3 $(n=1)$, programmed death ligand 1 (PDL1) $(n=1)$, human epidermal growth factor 2 (HER2)-NEU $(n=1)$, CD68 $(n=1)$, and mucicarmine $(n=1)$.

\section{Discussion}

This cohort study evaluated application of the recently proposed ISRSFC on reporting PF cytopathology. The study consisted of 299 cases, including $252 \mathrm{NFM}(84.3 \%)$, 30 MAL (10\%), 13 AUS (4.3\%), and 4 SFM (1.4\%) cases. In all, $96.6 \%$ of $\mathrm{PF}$ specimens received a definitive diagnosis as either benign or malignant, and only a small proportion of cases were diagnosed as AUS or SFM (5.7\%). The ratio of male to female was significantly low in all categories, 121/131 in NFM, 6/7 in AUS, and 7/23 in MAL, except for SFM which was higher in males than females (3/1). The mean age was 50 s for all diagnostic categories (51-58 years), with the lowest for MAL (51 years) and highest for SFM (58 years). The volume for all cases varied from 3 to $1,700 \mathrm{~mL}$. This study shows that volume has no impact on the diagnostic outcome of PF. NFM PEs composed $84.3 \%$ (252/300) of the cases, a percentage higher than that in other reported studies; however, this may be due to our patient population [16]. The second most common category was the MAL, comprising $10 \%$ of the cases. SFM was the smallest category, comprising only $1.4 \%$ of the cases. The diagnosis of SFM is used in our institution when there is a highly atypical cell population with cytomorphologic features suggestive of malignancy, but a definitive diagnosis cannot be achieved, mostly due to a low quantity of markedly atypical cells with the exception of cases with atypical lymphocytes. The number of atypical cells is variable but considered insufficient for a definitive diagnosis, given the implication of a diagnosis of stage IV disease. We do not use a specific number of cells to determine if a specimen is suspicious or positive for malignancy. In patients with a well-established history of disseminated malignancy, few highly atypical cells might be enough for the diagnosis of malignancy if supported by IHC. In other clinical scenarios, the same number of cells might not be sufficient for a diagnosis of malignancy. In all, 1.5\% $(90 / 6,178)$ of pleural effusion specimens were diagnosed as SFM in our recent study [17].

The most common risk factors seen in our patients with NFM PE were non-anticoagulant medications including chemotherapy $(73 / 252,29 \%)$; cardiovascular conditions $(44 / 252,17.4 \%)$ such as cardiac surgery, myocardial infarction, and ablation therapy; uremia (37/252, $14.7 \%)$; connective tissue disorders such as systemic lupus erythematosus $(27 / 252,10.7 \%)$; infections such as coxackie B virus (19/252, 7.5\%); and anticoagulant agents $(18 / 252,7.1)$. Less common causes of PE included hypothyroidism $(7 / 252,2.7 \%)$; organ transplant, both solid and bone marrow $(7 / 252,2.7 \%)$; radiation $(3 / 252,1.2 \%)$; and trauma $(3 / 252,1.2 \%)$.

Common cellular findings seen in NFM PF include mesothelial cells, lymphocytes, histiocytes, and RBCs. Interestingly, our study suggests that specimens where no mesothelial cells are seen can still be considered satisfactory for evaluation, since none of the patients developed MAL PE during at least 6 months of follow-up. Blood only was seen in 26 cases. In addition to cardiovascular conditions, kidney failure, connective tissue disorders, medications, and anticoagulant agents can contribute to a blood-only specimen. This finding has not been reported in previous published studies $[16,18]$. Hemosiderin-laden macrophages can occasionally be seen in benign PF. Hemosiderin-laden macrophages can also be seen in patients with underlying ESRD, hypothyroidism, cardiac amyloidosis, and anticoagulant therapy. Interestingly, the NFM category exhibited very variable associated clinical conditions, such as trauma, cardiomyopathy, malignancy, ESRD, and hypothyroidism. However, no correlation was found between patients' clinical condition and the cellular component of the PEs. Among infectious agents, bacterial infections were the most common conditions seen in PF, including Propionibacterium acnes $(n=3)$, Propionibacterium acnes and Streptococcus viridans $(n=1)$, Streptococcus pneumoniae ( $n=$ 2 ), and methicillin-resistant Staphylococcus aureus ( $n=$ $2)$. African trypanosomiasis was a rare finding $(n=1)$. None of our cases showed mycobacterial infections, while Mycobacterium tuberculosis, Mycobacteruim avi- 
um complex, and Mycobacterium kansasii were reported previously $[8,16]$. Our study revealed coxsackie B virus $(n=1)$ in PF. Parvovirus B19, hepatitis C virus, influenza virus, adenovirus, and Enterovirus were reported previously [8]. African trypanosomiasis was detected in one of our cases. African trypanosomiasis may cause cardiomyopathy and PE [19].

Almost $10 \%$ of all PF samples were diagnosed as MAL $(30 / 299)$. This is a much lower percentage than in previous studies $[16,18]$. One study of PE found $23 \%$ of MAL, although their patient population included only those admitted to the hospital [7]. Our study included both outpatient and hospitalized patients. The majority of the patients in our study were females $(n=23,76.7)$ with mean age of 51 years. Secondary malignancies were the most common cause of MAL PE. Lung cancer (11/30, including 8 lung adenocarcinomas, 2 small cell lung cancer, and 1 squamous cell carcinoma) followed by breast cancer and hematopoietic malignancies were the most common causes of malignant PE in our study, which is similar to a previous study [16], while breast cancer was reported as the most common primary site in other prior published studies $[16,20]$. Lung adenocarcinoma was the most common type of lung cancer involving PF in our study, while small cell lung cancer was reported to be the most common type of lung cancer in other studies [16]. Interestingly, our prior study showed that lung cancer followed by breast cancer were the most common secondary malignancies in pleural effusion as well [14]. Carcinomas of the gastrointestinal tract were also primarily detected in $\mathrm{PF}$, including gastric carcinoma $(n=2)$ and colonic adenocarcinoma $(n=2)$. Acute lymphotic leukemia (ALL) involved PF in 2 cases. Uncommon malignant neoplasms involving PEs were metastatic NUT carcinoma, ovarian carcinoma, renal cell carcinoma, metastatic rhabdomyosarcoma, T-cell lymphoma, and multiple myeloma [16].

Interpretation of PF on cytologic material can be challenging [21]. Extremely low or high ratio of malignant cells to mesothelial cells and single-cell-pattern distribution of malignant cells are all considered common causes of diagnostic errors [21]. Therefore, IHC can be used to improve diagnostic accuracy. Immunostains were performed on 28 cases $(9.4 \%)$, including 15 MAL cases, 10 AUS cases, and 3 SFM cases. Application of immunostains resulted in a definitive diagnosis in 15 MAL cases. Although immunostains were attempted in 13 of 17 inconclusive cases, including both AUS and SFM cases, the immunostains were noncontributory due to scant cellularity. In these circumstances, collection of additional flu- id may improve the diagnosis. Our study shows that the presence of mesothelial cells is not necessary for specimen adequacy and PF may consist of blood only. This finding has not been reported in prior studies. Moreover, $\mathrm{PF}$ volume is not a predictive factor for the diagnosis of malignancy.

In conclusion, the ISRSFC is a user-friendly reporting system, which is easily applicable on serous effusions including PEs. The majority of PE cases fall in the NFM category (84.3\%). MAL cases comprise a relatively small proportion of PEs (10\%) with a significant clinical impact. In all, $94.3 \%$ of cases carried a definitive diagnosis (NFM and MAL). Cases with indefinite diagnosis constituted less than $6 \%$ of all cases (AUS and SFM). Our study shows that the presence of mesothelial cells is not necessary for specimen adequacy in PEs. PF specimens can present as blood only in variable clinical conditions.

\section{Statement of Ethics}

The study protocol has been approved by the research institute's Committee on Human Research.

\section{Disclosure Statement}

The authors have no financial conflict of interest to disclose.

\section{Funding Sources}

The authors did not receive any funding.

\section{Prior Presentation}

This work was presented as a poster at the 67th ASC annual meeting in Salt Lake City, Utah, in November 2019.

Erika Rodriguez: conceptualization, methodology, validation, formal analysis, investigation, resources, data curation, writing original draft, visualization, supervision, and project administration. Ricardo Pastorello, Robert Jones, and Mathew Gabrileson: data curation, writing - review. Dustin Santos: data curation. Zahra Maleki: conceptualization, methodology, validation, formal analysis, investigation, resources, data curation, writing - review and editing, visualization, supervision, and project administration. 


\section{References}

1 Nayar R, Wilbur DC, editors. The bethesda system for reporting cervical cytology: definitions. 3rd ed. New York, USA; Cham, Switzlerland: Springer Press; 2015. ISBN-13: 9783319110738.

2 Ali S, Cibas E, editors. The Bethesda system for reporting thyroid cytopathology, criteria and explanatory notes. New York: Springer; 2018.

3 Wojcik E, Kurtycz D, editors. The paris system for reporting urinary cytology. New York; Cham, Switzlerland: Springer Press; 2016. ISBN 978-3-319-22863-1.

4 Faquin WC, Rossi ED, Baloch Z, Barkan GA, Foschini MP, Kurtycz DFI, editors. The milan system for reporting salivary gland cytopathology. Cham, Switzerland: Springer Press; 2018. ISBN 978-3-319-71284-0..

5 Chandra A, Crothers B, Kurtycz D, Schmitt F. Announcement: the international system for reporting serous fluid cytopathology. Acta Cytol. 2019;63(5):349-51.

6 Hoit BD. Pericardial effusion and cardiac tamponade in the new millennium. Curr Cardiol Rep. 2017;19(7):57.

7 Corey GR, Campbell PT, Van Trigt P, Kenney RT, O'Connor CM, Sheikh KH, et al. Etiology of large pericardial effusions. Am J Med. 1993; 95(2):209-13.

8 Levy PY, Corey R, Berger P, Habib G, Bonnet JL, Levy S, et al. Etiologic diagnosis of 204 pericardial effusions. Medicine. 2003;82(6): 385-91.
9 Strobbe A, Adriaenssens T, Bennett J, Dubois C, Desmet W, McCutcheon K, et al. Etiology and long-term outcome of patients undergoing pericardiocentesis. J Am Heart Assoc. 2017;6(12):pii: e007598. 10.1161/ JAHA.117.007598.

10 Wilkes JD, Fidias P, Vaickus L, Perez RP. Malignancy-related pericardial effusion. 127 cases from the roswell park cancer institute. Cancer. 1995;76(8):1377-87.

11 Klatt EC, Heitz DR. Cardiac metastases. Cancer. 1990;65(6):1456-9.

12 Adler Y, Charron P, Imazio M, Badano L, Barón-Esquivias G, Bogaert J, et al. 2015 ESC guidelines for the diagnosis and management of pericardial diseases: the task force for the diagnosis and management of pericardial diseases of the European society of cardiology (ESC) endorsed by: the European association for cardio-thoracic surgery (EACTS). Eur Heart J. 2015;36(42):2921-64.

13 Carter J, Miller JA, Feller-Kopman D, Ettinger D, Sidransky D, Maleki Z. Molecular profiling of malignant pleural effusion in metastatic non-small-cell lung carcinoma. The effect of preanalytical factors. Ann Am Thorac Soc. 2017;14(7):1169-76.

14 Rodriguez EF, Chowsilpa S, Maleki Z. The differential diagnosis in nonlymphoproliferative malignant pleural effusion cytopathology and its correlation with patients' demographics. Acta Cytol. 2018;62(5-6):436-42.
15 Rodriguez EF, Shabihkhani M, Carter J, Maleki Z. Molecular alterations in patients with pulmonary adenocarcinoma presenting with malignant pleural effusion at the first diagnosis. Acta Cytol. 2017;61(3):214-22.

16 Dragoescu EA, Liu L. Pericardial fluid cytology: an analysis of 128 specimens over a 6-year period. Cancer Cytopathol. 2013; 121(5):242-51.

17 Rodriguez E, Pastorello R, Chowsilpa S, Maleki Z. Suspicious for malignancy diagnoses on pleural effusion cytology. J Am Soc Cytopathol. 2019;8(5):S14.

18 Yadav S, Singh M, Singh P, Mandal S, Khurana N, Jain SL. Cytological evaluation of pericardial fluids: a 5 years experience in tertiary care center. Indian J Pathol Microbiol. 2019; 62(2):270-3.

19 Xiao Z, Dong A, Wang Y. FDG PET/CT in a case of human african trypanosomiasis (sleeping sickness). Clin Nucl Med. 2018; 43(8):619-22.

20 Vakamudi S, Ho N, Cremer PC. Pericardial effusions: causes, diagnosis, and management. Prog Cardiovasc Dis. 2017;59(4): 380-8.

21 Moriarty AT, Stastny J, Volk EE, Hughes JH, Miller TR, Wilbur DC, et al. Fluids-good and bad actors: observations from the college of American pathologists interlaboratory comparison program in nongynecologic cytology. Arch Pathol Lab Med. 2004;128(5):513-8. 